\title{
Belphégor
}

\section{Dumas, Alexandre, Correspondance générale, tome II, édition de Claude Schopp}

\section{Vittorio Frigerio}

\section{(2) OpenEdition}

1 Journals

\section{Electronic version}

URL: http://journals.openedition.org/belphegor/946

DOI: 10.4000/belphegor.946

ISSN: 1499-7185

\section{Publisher}

LPCM

\section{Electronic reference}

Vittorio Frigerio, «Dumas, Alexandre, Correspondance générale, tome II, édition de Claude Schopp », Belphégor [Online], 15-2 | 2017, Online since 02 November 2017, connection on 21 December 2020. URL : http://journals.openedition.org/belphegor/946 ; DOI : https://doi.org/10.4000/belphegor.946

This text was automatically generated on 21 December 2020.

\section{(c) (i) (9)}

Belphégor est mis à disposition selon les termes de la Licence Creative Commons Attribution - Pas d'Utilisation Commerciale - Pas de Modification 4.0 International. 


\title{
Dumas, Alexandre, Correspondance générale, tome II, édition de Claude Schopp
}

\author{
Vittorio Frigerio
}

\section{REFERENCES}

Dumas, Alexandre. Correspondance générale. Tome II. Édition de Claude Schopp. Paris :

Classiques Garnier, 2016. 775 p.

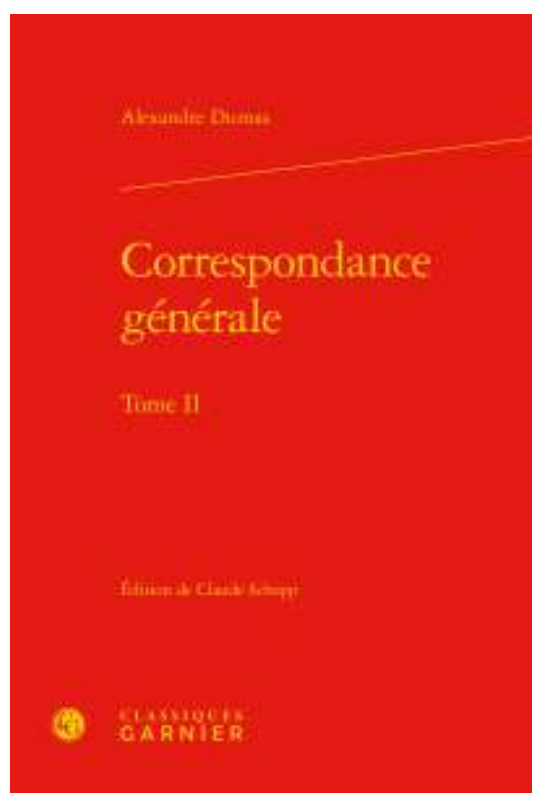

1 Claude Schopp poursuit son grand travail d'édition de la correspondance du plus prolifique des écrivains du dix-neuvième siècle français avec ce nouveau tome, qui va du début 1833 à août 1838, période, nous dit-il, de « désillusion », « années horribles » 
pendant lesquelles les grands espoirs et les enthousiasmes de la jeunesse se heurtent à des déceptions de toutes sortes, et qui finit avec la mort de la mère tant aimée. Mais si ces années ont réservé des surprises guère toujours agréables à l'auteur, le lecteur aura tôt fait de se rendre compte qu'elles n'ont pas affecté son style, entraînant et vigoureux même dans les rapports les plus ordinaires. On redécouvre en parcourant ces pages la dispute qui opposa Dumas et Hugo au sujet de l'article de Granier de Cassagnac publié dans le Journal des débats politiques et littéraires - article par ailleurs heureusement reproduit en annexe, ce qui permet de juger sur pièces des accusations et des insinuations que ce protégé du grand Victor pouvait se croire habilité à porter sur le grand Alexandre. Au fil des pages brillent aussi avec une vigueur et une chaleur que le passage du temps n'a pas su affecter, les lettres, toujours pressantes, toujours exaltées, de Marie Dorval, fenêtres idéales sur l'âme aimante et emportée de cette actrice qui fit le succès d'Antony et de tant d'autres pièces importantes de Dumas et des premières plumes du romantisme. A part le menu fretin de la correspondance journalière (détails de contrats, lettres aux et des éditeurs et autres écrits de ce genre, qui sauront sans doute être utiles aux chercheurs et aux spécialistes), le plaisir que l'on a à parcourir ce volume vient principalement de l'impression de vie fougueuse qui s'en dégage, du style de la plus petite bafouille (qui a de quoi souffler totalement tout habitué contemporain de Twitter et de la communication élémentaire par courrier électronique), de la beauté esthétique de ces messages souvent pourtant ordinaires, dans lesquels les besoins de l'information ne prennent jamais le dessus sur le plaisir de construire de belles phrases, de donner à tout moment à sa pensée une forme qui suscite le plaisir et l'admiration. Ce sont de véritables personnages qui revivent dans l'esprit du lecteur en relisant les bouillants échanges avec Gaillardet, longs, pénibles et compliqués, au sujet du drame de La Tour de Nesle, ou les rapports inénarrables entre Dumas et sa diverses maîtresses, amantes ou admiratrices, ou encore les plans, malheureusement voués, en tout ou en partie, à l'échec, pour son grand voyage autour de la Méditerranée ou pour la création d'un nouveau théâtre. Et de temps à autre apparaissent quelques déclarations fulgurantes de simplicité et de justesse, qui situent l'homme, le cernent, le font mieux comprendre. Et cela que ce soit pour ce qui est des rapports humains ( « [...] ce que vous voulez bien appeler la gloire, n'a toujours été pour moi qu'un moyen d'arriver au bonheur, et mon bonheur à moi c'est dans l'amour que je l'ai constamment cherché et malheureusement sans le trouver jamais [...]» [210]) ou en ce qui concerne sa vision de l'art (« [...] la seule manière de critiquer est d'entrer dans son système, de s'enfermer dans le plan qu'il s'est tracé, et de le juger dans le drame qu'il a fait, et non dans celui qu'un autre aurait pu faire : le talent, n'est talent que s'il est individuel. Il faut donc l'accepter dans son individualité. » [126]).

2 Comme pour le premier tome, le lecteur a droit pour chaque année à une chronologie fort utile Après les Annexes (parmi lesquels il faut encore signaler l'article «Curiosités et monuments historiques. La Tour de Nesle», publié par Frédéric Gaillardet dans le Musée des familles en 1834) figurent également un répertoire des correspondants, et toute une série d'index (des noms de personnes, des lieux, des œuvres citées, des personnages littéraires cités, des journaux et périodiques cités, des œuvres picturales citées) qui font de ce volume un ouvrage de référence particulièrement facile et agréable à utiliser. Rien n'étant jamais parfait, les lecteurs italophones ne pourront réprimer une certaine gêne en voyant des transcriptions de lettres en cette langue dans lesquelles figurent de nombreuses fautes. Si on peut présumer que celles-ci peuvent être le fait de l'auteur lorsqu'il s'agit de petites phrases jetées ici et là pour «faire 
chic », comme dans la correspondance de Virginie Bourbier (une actrice proche de Dumas), cela paraît bien moins probable lorsqu'elles sont, par exemple, de la plume de quelque ministre du Royaume des Deux-Siciles. Heureusement, la traduction en français, fournie en note, vient éclaircir tous les mystères.

\section{AUTHOR}

\section{VITTORIO FRIGERIO}

Dalhousie University 